\title{
The Gluon Sivers Distribution: Status and Future Prospects
}

\author{
Daniël Boer, ${ }^{1}$ Cédric Lorcé, ${ }^{2,3}$ Cristian Pisano, ${ }^{4}$ and Jian $Z_{h o u}{ }^{5}$ \\ ${ }^{1}$ Van Swinderen Institute, University of Groningen, Nijenborgh 4, 9747 AG Groningen, Netherlands \\ ${ }^{2}$ SLAC National Accelerator Laboratory, Stanford University, Menlo Park, CA 94025, USA \\ ${ }^{3} I F P A$, AGO Department, University of Liège, Sart-Tilman, 4000 Liège, Belgium \\ ${ }^{4}$ Department of Physics, University of Antwerp, Groenenborgerlaan 171, 2020 Antwerp, Belgium \\ ${ }^{5}$ Nikhef and Department of Physics and Astronomy, VU University Amsterdam, De Boelelaan 1081, 1081 HV Amsterdam, Netherlands \\ Correspondence should be addressed to Daniël Boer; d.boer@rug.nl
}

Received 16 April 2015; Accepted 28 June 2015

Academic Editor: Jibo He

Copyright (C) 2015 Daniël Boer et al. This is an open access article distributed under the Creative Commons Attribution License, which permits unrestricted use, distribution, and reproduction in any medium, provided the original work is properly cited. The publication of this article was funded by SCOAP S $^{3}$

\begin{abstract}
We review what is currently known about the gluon Sivers distribution and what are the opportunities to learn more about it. Because single transverse spin asymmetries in $p^{\uparrow} p \rightarrow \pi X$ provide only indirect information about the gluon Sivers function through the relation with the quark-gluon and tri-gluon Qiu-Sterman functions, current data from hadronic collisions at RHIC have not yet been translated into a solid constraint on the gluon Sivers function. SIDIS data, including the COMPASS deuteron data, allow for a gluon Sivers contribution of natural size expected from large $N_{c}$ arguments, which is $\mathscr{O}\left(1 / N_{c}\right)$ times the nonsinglet quark Sivers contribution. Several very promising processes to measure the gluon Sivers effect directly have been suggested, which besides RHIC investigations, would strongly favor experiments at AFTER@LHC and a possible future Electron-Ion Collider. Due to the inherent process dependence of TMDs, the gluon Sivers TMD probed in the various processes are different linear combinations of two universal gluon Sivers functions that have different behavior under charge conjugation and that therefore satisfy different theoretical constraints. For this reason both hadronic and DIS type of collisions are essential in the study of the role of gluons in transversely polarized protons.
\end{abstract}

\section{The Sivers Function and Its Definition}

The distribution of quarks and gluons in a proton (or any other spin- $1 / 2$ hadron) that is polarized transversely to its momentum need not be left-right symmetric with respect to the plane spanned by the momentum and spin directions. This asymmetry is called the Sivers effect [1]. It results in angular asymmetries of produced particles in high energy scattering processes involving a transversely polarized hadron. Experimental data in support for such a left-right asymmetry in the quark distribution was first obtained from semi-inclusive DIS process by the HERMES collaboration [2]. This review is about what is currently known about the gluonic Sivers effect distribution. The Sivers effect is of great interest theoretically as it is very sensitive to the color flow in the scattering process and to the multitude of color exchanges among initial and final states. It is the first quantity for which this has been recognized and for which color flow sensitivity can be tested unambiguously [3]. Verification of its unusual properties will provide a strong test of the formalism of Transverse-Momentum-Dependent parton distributions. It is an important quantity of nonperturbative QCD to consider both qualitatively and quantitatively. This review discusses these aspects for the gluon Sivers distribution specifically. We first start with its proper definition.

The number density in momentum space of a generic parton (quark, antiquark, and gluon) inside a hadron with mass $M$, transverse polarization $\mathbf{S}_{T}$, and momentum $\mathbf{P}$ can be written as

$$
\widehat{f}\left(x, \mathbf{k}_{\perp} ; \mathbf{S}_{T}\right)=f_{1}\left(x, \mathbf{k}_{\perp}^{2}\right)-\frac{\left(\widehat{\mathbf{P}} \times \mathbf{k}_{\perp}\right) \cdot \widehat{\mathbf{S}}_{T}}{M} f_{1 T}^{\perp}\left(x, \mathbf{k}_{\perp}^{2}\right),
$$

where $f_{1}\left(x, \mathbf{k}_{\perp}^{2}\right)$ is the unpolarized Transverse-MomentumDependent (TMD) parton distribution, $\widehat{\mathbf{S}}_{T} \equiv \mathbf{S}_{T} /\left|\mathbf{S}_{T}\right|$, and $\widehat{\mathbf{P}} \equiv \mathbf{P} /|\mathbf{P}|$. The function $f_{1 T}^{\perp}\left(x, \mathbf{k}_{\perp}^{2}\right)$ describing the distortion 
in the distribution of unpolarized partons with light-front momentum fraction $x$ and transverse momentum $\mathbf{k}_{\perp}$ due to the transverse polarization of the hadron is called the Sivers function. The notation used here comes from [4], but also the notation $\Delta^{N} f_{g / h^{\uparrow}}$ from $[5,6]$ is sometimes used, where $\Delta^{N} f_{g / h^{\uparrow}}=-2\left(\left|\mathbf{k}_{\perp}\right| / M\right) f_{1 T}^{\perp g}$ (analogous to the quark case [7]).

The Sivers function satisfies the following positivity bound [8]:

$$
\frac{\left|\mathbf{k}_{\perp}\right|}{M}\left|f_{1 T}^{\perp}\left(x, \mathbf{k}_{\perp}^{2}\right)\right| \leq f_{1}\left(x, \mathbf{k}_{\perp}^{2}\right) .
$$

In [8] the operator definition of the gluon Sivers function, which was called $G_{T}=-f_{1 T}^{\perp g}[9]$, was first given without gauge links. The definition including gauge links then appeared in $[10,11]$

$$
\begin{aligned}
- & \frac{\left(\widehat{\mathbf{P}} \times \mathbf{k}_{\perp}\right) \cdot \widehat{\mathbf{S}}_{T}}{M} f_{1 T}^{\perp g}\left(x, \mathbf{k}_{\perp}^{2}\right) \\
& =\frac{1}{2}\left[\widehat{f}\left(x, \mathbf{k}_{\perp} ; \mathbf{S}_{T}\right)-\widehat{f}\left(x, \mathbf{k}_{\perp} ;-\mathbf{S}_{T}\right)\right]
\end{aligned}
$$

with

$$
\begin{gathered}
\widehat{f}\left(x, \mathbf{k}_{\perp} ; \mathbf{S}_{T}\right)=\frac{\delta_{T}^{j l}}{x P^{+}} \int \frac{\mathrm{d} z^{-} \mathrm{d}^{2} z_{\perp}}{(2 \pi)^{2}} e^{i k \cdot z}\left\langle P, S_{T}\right| 2 \\
\left.\cdot \operatorname{Tr}\left[F^{+j}(0) U_{[0, z]} F^{+l}(z) U_{[z, 0]}\right]\left|P, S_{T}\right\rangle\right|_{z^{+}=0},
\end{gathered}
$$

where $U_{[a, b]}$ is a Wilson line connecting the points $a$ and $b$ along a contour determined by the physical process and $k^{+}=x P^{+}$is the fraction of parton light-front momentum. For a proper definition that is free from rapidity divergences associated with gauge links with paths (partly) along the light front, a redefinition involving the so-called soft factor is necessary [12-15]. This will however not play a significant role here and can simply be considered as implicit.

\section{Sivers Effect, $A_{N}$, and Qiu-Sterman Effect}

The Sivers effect (for both quarks and gluons) was first suggested in [1] as an explanation for the large left-right single transverse spin asymmetries $\left(A_{N}\right)$ observed in $p^{\uparrow} p \rightarrow \pi X$ [16-23] (and similar asymmetries in $K$ [22], in $\eta$ [24], and tentatively in $J / \psi$ [25] production). The Sivers effect was first studied phenomenologically in [5]. However, extraction of the Sivers TMD presumes all-order TMD factorization. A factorized description of the process $p p \rightarrow \pi X$ only applies for large transverse momentum $p_{T}$ of the produced pions (say for $p_{T} \gtrsim 1 \mathrm{GeV}$ ), where in fact collinear factorization is appropriate [26], rather than TMD factorization. Although TMDs do appear in the phenomenological description of $A_{N}$ in [5] and subsequent studies (see [27]), that description is thus not based on a TMD factorization theorem. Rather it should be considered as an effective model description, now commonly referred to as the Generalized Parton Model (GPM). As a phenomenological approach it has proven useful in the quest to disentangle the possible underlying mechanisms of the spin asymmetries; see [27, 28] for more discussion, but the extracted "effective" TMDs may differ from the TMDs extracted from TMD-factorizing processes. What is known about the effective gluon Sivers TMD will be discussed below.

In collinear factorization the single spin asymmetry (SSA) will arise at the twist-3 level [29]. In this description $A_{N}$ probes the (quark-gluon) Qiu-Sterman function (sometimes one factor of the coupling constant $g$ is included in the definition of Qiu-Sterman functions, because one always encounters them multiplied by $g$ ) $[30,31]$

$$
\begin{gathered}
T_{q, F}(x, x)=\frac{M}{P^{+}} \int \frac{\mathrm{d} z^{-} \mathrm{d} \eta}{2 \pi} e^{i k \cdot z} \frac{\left(\widehat{\mathbf{P}} \times \widehat{\mathbf{S}}_{T}\right)^{j}}{2 M}\langle P, S| \bar{\psi}(0) \\
\left.\cdot \gamma^{+} F^{+j}(\eta z) \psi(z)|P, S\rangle\right|_{z^{+}=\left|\mathbf{z}_{\perp}\right|=0},
\end{gathered}
$$

and its trigluon correlation analogues $T_{G}^{(f)}(x, x)=T_{G}^{(+)}(x, x)$ and $T_{G}^{(d)}(x, x)=T_{G}^{(-)}(x, x)[32-35]$

$$
T_{G}^{( \pm)}(x, x)=-\frac{2 M \delta_{T}^{l m}}{x\left(P^{+}\right)^{2}} \int \frac{\mathrm{d} z^{-} \mathrm{d} \eta}{2 \pi} e^{i k \cdot z} \frac{\left(\widehat{\mathbf{P}} \times \widehat{\mathbf{S}}_{T}\right)^{j}}{2 M}\langle P, S|
$$

$$
\left.\cdot C_{ \pm}^{a b c} F_{a}^{+l}(0) F_{b}^{+j}(\eta z) F_{c}^{+m}(z)|P, S\rangle\right|_{z^{+}=\left|\mathbf{z}_{\perp}\right|=0},
$$

where $C_{+}^{a b c}=i f^{a b c}$ and $C_{-}^{a b c}=d^{a b c}$ and where the light-front gauge $A^{+}=0$ has been considered for convenience.

In [36] the quark-gluon Qiu-Sterman function has been related to the first transverse moment of the quark Sivers function, that is, $f_{1 T}^{\perp(1) q}(x) \propto T_{q, F}(\mathrm{x}, x) / M$, where

$$
f_{1 T}^{\perp(1) q}(x) \equiv \int \mathrm{d}^{2} k_{\perp} \frac{\mathbf{k}_{\perp}^{2}}{2 M^{2}} f_{1 T}^{\perp q}\left(x, \mathbf{k}_{\perp}^{2}\right) .
$$

However, that relation was only established at tree level (beyond tree level the relation will be affected by the considered regularized definition of the Sivers TMD including its dependence on the soft factor). A similar tree level relation can be established in the gluon sector as well: $f_{1 T}^{\perp(1) g}(x) \propto$ $T_{G}(x, x) / M$ (where $T_{G}$ that appears in this relation depends on the gauge links; see the discussion in Section 5).

Another relation has been established in [37]:

$$
f_{1 T}^{\perp q}\left(x, \mathbf{k}_{\perp}^{2}\right) \stackrel{\mathbf{k}_{\perp}^{2} \gg M^{2}}{\sim} \alpha_{s} \frac{M}{\mathbf{k}_{\perp}^{4}}\left(K \otimes T_{q, F}\right)(x),
$$

which means that the quark-gluon Qiu-Sterman function determines the large transverse momentum tail of the quark Sivers function. Here it should be emphasized that the function $\left(K \otimes T_{q, F}\right)(x)$ consists not only of a convolution of $T_{q, F}(x, x)$ but also of its derivative $x \partial T_{q, F}(x, x) / \partial x$ and of the more general $T_{q, F}(x, y)$ with $y \neq x$. It corresponds to the fact that the evolution of $T_{q, F}$ is nonautonomous and inhomogeneous; see [38]. Note that here we have discussed the nonsinglet contributions only that apply to combinations like $u$ minus $d$ quarks; otherwise also gluonic contributions need to be taken into account. 
Similarly, the tail of the gluon Sivers function is determined by several Qiu-Sterman functions [39]. It receives contributions from the quark-gluon Qiu-Sterman functions $T_{q, F}(x, x)$ and $T_{q, F}(x, y)$ with $y \neq x$ and from the trigluon functions $T_{G}^{(f / d)}(x, x)$ (whose contribution to the gluon Sivers function depends on the gauge links; see Section 5). At small $x$ the situation simplifies: the contributions from $T_{q, F}(x, x)$ and $T_{q, F}(x, y)$ with $y \neq x$ to the tail of the gluon Sivers function cancel each other [39], leaving only the trigluon correlators. Moreover, $T_{G}^{(d)}(x, x)$ evolves with the same $1 / x$ behavior at small $x$ as the unpolarized gluon distribution and is therefore not necessarily suppressed at high energies and small values of $x$, whereas $T_{G}^{(f)}(x, x)$ lacks this $1 / x$ enhancement [35]. As the large $p_{T} A_{N}$ data from RHIC are generally not in the small- $x$ region of the polarized proton, except for negative $x_{F}$, any simplifications at small $x$ should of course first be tested for validity.

Information from $A_{N}$ measurements at sufficiently large $p_{T}$ (in order to consider a collinear factorization description in the first place) can thus in principle reflect some information on Sivers functions (i.e., on the tails and perhaps also on first transverse moments), but in practice other twist-3 contributions beside the mentioned Qiu-Sterman functions, namely, chiral-odd and fragmentation function analogues, contribute to $A_{N}[28,40-42]$. From the smallness of $A_{N}$ in the midrapidity and backward (negative $x_{F}$ ) regions, one would generally conclude that gluonic and sea quark contributions to the transverse single spin asymmetry are not large, but a detailed analysis is required to determine precisely the size of the various contributions.

$A_{N}$ for $\pi^{0}$ production at midrapidity has been measured by the PHENIX experiment in polarized $p p$ collisions at RHIC and was found to be consistent with zero, for $p_{T}$ values below $5 \mathrm{GeV}$ at the permille level and for higher $p_{T}$ values (up to $11 \mathrm{GeV}$ ) at the few percent level $[21,43]$. These data taken at $\sqrt{s}=200 \mathrm{GeV}$ probe $x$ values only down to $x \sim 0.006$, where still a combination of Qiu-Sterman functions is expected to contribute. In [44] these $\pi^{0}$ data were discussed, using two models for the trigluon Qiu-Sterman functions that were constrained from $A_{N}$ in $D$-meson production $[45,46]$. The midrapidity $\pi^{0}$ data are shown to mostly constrain $T_{G}^{(f)}(x, x)$ (their $N(x)$ ) at low $p_{T}$. The authors conclude that "both models give tiny asymmetry due to the small partonic cross sections, so the form of the three-gluon correlation functions is not much constrained by the data in this region." From this limited model study of both $\pi^{0}$ and $D$ production one would conclude that $T_{G}^{(f)}(x, x)$ and $T_{G}^{(d)}(x, x)$ are in any case small, a permille fraction of $x$ times the unpolarized gluon distribution. This should be investigated further with more general model forms that adhere to the correct small- $x$ behavior and with more precise data. The experimental precision of $A_{N}$ can be improved much further both at RHIC and especially at the AFTER@LHC experiment [47], which would have a luminosity factor of 10-100 times higher, if not more. Such improvement and the measurements of asymmetries for many different types of produced particles are required to separately constrain or determine the various Qiu-Sterman contributions.
In the GPM the smallness of $A_{N}$ at midrapidity puts strong constraints on the effective gluon Sivers function. As explained, this gluon Sivers function captures the combined effect from several Qiu-Sterman contributions and may thus differ from the actual gluon Sivers function obtained from TMD-factorizing processes. In a recent GPM analysis [48], which is an updated analysis of [49], the best fits to the PHENIX midrapidity $\pi^{0} A_{N}$ data indeed correspond to a small effective gluon Sivers function with respect to its theoretical bound determined by the unpolarized gluon. For example, for $x<0.1$ it is at most only a few percent of the bound. However, the maximally allowed effective gluon Sivers function is still sizable though. Its first transverse moment is still found to be around $30 \%$ of the up quark Sivers function in the region $0.06<x<0.3$, which is consistent with findings from semi-inclusive DIS for the actual gluon Sivers function and also with theoretical expectations, as discussed in the next section. In addition, it should be mentioned that this GPM analysis assumes a Gaussian $k_{\perp}$ dependence, which corresponds neither to the correct power-law tail of the Sivers function, (8), nor to the unpolarized gluon distribution. Given all the caveats that come with these results, one should be careful to draw a definite conclusion about the size of the actual gluon Sivers TMD from $A_{N}$ data.

One should also specify clearly what one calls a small gluon Sivers function. It will depend on what one compares to, that is, whether that is to the unpolarized gluon that grows very rapidly at small $x$ or to the up or down quark Sivers function for not too small $x$. At small $x$ it becomes very important whether one discusses the $f$ or $d$ type contribution (see Section 5), which is an issue not addressed in the GPM studies of $A_{N}$.

\section{Sivers Asymmetry in SIDIS}

The Sivers effect leads to a $\sin \left(\phi_{h}-\phi_{S}\right)$ asymmetry in semi-inclusive DIS (SIDIS) [4], which has been observed in experiments using a proton target by HERMES [2, 50] and COMPASS [51] and using ${ }^{3} \mathrm{He}$ target by Jefferson Lab Hall A $[52,53]$. The data follow to quite a good extent the expectations of a valence quark picture in the target and of favored fragmentation. In the proton case, $\pi^{+}$thus shows the largest asymmetry (for large $z$ values, the asymmetry is around $4-5 \%$ or even somewhat larger when a lower cut of $Q^{2}>4 \mathrm{GeV}^{2}$ is implemented instead of $\left.Q^{2}>1 \mathrm{GeV}^{2}[50]\right)$. The $\pi^{-}$asymmetries are smaller and still compatible with zero. The $K^{ \pm}$asymmetries are similar to the $\pi^{ \pm}$asymmetries, but with larger errors. Sivers asymmetries on a deuteron target [54] are all consistent with zero. Fits to all these HERMES and COMPASS data, including the deuteron data using isospin symmetry, indicate that the Sivers function $\left(f_{1 T}^{\perp}\right)$ for $u$ quarks in a proton is negative and for a $d$ quark in a proton is positive and approximately equal in absolute value [55]. This fits the expectations from the limit of a large number of colors $N_{c}[56,57]$ :

$$
f_{1 T}^{\perp u}\left(x, \mathbf{k}_{\perp}^{2}\right)=-f_{1 T}^{\perp d}\left(x, \mathbf{k}_{\perp}^{2}\right)+\mathcal{O}\left(\frac{1}{N_{c}}\right) .
$$


The flavor singlet combination of $u$ and $d$ is of the same order as the gluon contribution in $N_{c}$ counting [58]. The latter is thus $1 / N_{c}$ suppressed with respect to the flavor nonsinglet quark Sivers effect at not too small $x\left(x \sim 1 / N_{c}\right)$ [59].

Within the current accuracy, the SIDIS data do not require any sea quark or gluon contributions, which among other considerations (see Section 6) led Brodsky and Gardner to conclude that the gluon Sivers function is small or even zero ("absence of gluon orbital angular momentum") [60]. The SIDIS data from HERMES, COMPASS, and Jefferson Lab Hall A are of course at rather modest $Q^{2}$ and not too small- $x$ values, that is, in the valence region. One cannot yet draw any conclusions about the gluon Sivers function at higher $Q^{2}$ and smaller values of $x$. Moreover, the data certainly still allow for gluon Sivers contributions of the order of $1 / N_{c}$ times the valence quark Sivers functions. This is evident from the fits by Anselmino et al. [55], where the first transverse moment of the $u$ and $d$ Sivers functions has error bands that are at least around $30 \%$ of the central values.

Note that the SSA in the "inclusive" process ep $\rightarrow h X$, where the back-scattered lepton is not observed $[61,62]$, does not allow for an interpretation in terms of TMDs, as the data are dominated by $Q^{2} \approx 0$. Even for large $p_{T}$ the appropriate factorization would be collinear factorization and the Sivers type of asymmetry would probe the Qiu-Sterman functions instead [63], which as discussed above have some relation to the Sivers TMDs, but only via the tail or possibly via the first transverse moment. The asymmetries for $p_{T}>1 \mathrm{GeV}$ are found to be at the level of $5-10 \%$ for positive hadrons. Fits will need to make clear how much room there is for a gluon Qiu-Sterman effect. Given the fact that the gluon QiuSterman function does not enter at leading order in $\alpha_{s}$ in this process, this room may be considerable.

\section{Sivers Asymmetry in Other Processes}

Several other $p p$ scattering processes to access the gluon Sivers function have been suggested over the past years: $p^{\uparrow} p \rightarrow \operatorname{jet} j e t X[64], p^{\uparrow} p \rightarrow D X[34,44,65], p^{\uparrow} p \rightarrow \gamma X$ [66], $p^{\uparrow} p \rightarrow \gamma$ jet $X[66,67], p^{\uparrow} p \rightarrow \gamma^{*} X \rightarrow \mu^{+} \mu^{-} X$ [66], $p^{\uparrow} p \rightarrow$ jet $X$ (single transverse spin asymmetries in jet production measured at RHIC $[68,69]$ at forward rapidities (the valence region) show very small asymmetries, which is probably due to a cancellation among $u$ and $d$ quark contributions [70]), $p^{\uparrow} p \rightarrow \pi$ jet $X$ [71], and $p^{\uparrow} p \rightarrow \eta_{c / b} X$ [39]. Several of these processes are like $A_{N}$ in (high- $p_{T}$ ) pion production, which means that they deal with twist-3 collinear factorization and only provide indirect or limited information about the gluon Sivers TMD. Several other processes run into the problem of TMD factorization breaking contributions [72] and hence are not safe. In principle they do probe TMDs but as a result of TMD factorization breaking contributions, conclusions about the gluon Sivers function from their measurements cannot be drawn safely. This applies, for instance, to the process $p^{\uparrow} p \rightarrow$ jet jet $X$ (measured at RHIC to be small at the few percent level [73]), which moreover suffers from cancellations between $u$ and $d$ contributions and between the effects of initial and final state interactions [74-76]. TMD factorization breaking would also apply to open heavy quark production: $p^{\uparrow} p \rightarrow Q \bar{Q} X$, such as $p^{\uparrow} p \rightarrow D^{0} \bar{D}^{0} X$; compare, for example, [77]. Whether the problem also applies to double heavy quarkonium production remains to be seen, because in practice the color singlet contributions may give the dominant contribution in that case. Among the hadronic collisions the processes having one or two color singlets in the final state would in any case be the safest. One very promising example is $p^{\uparrow} p \rightarrow \gamma$ jet $X$ [67], where it depends on the rapidity of the photon and the jet, that is, on the $x$ fraction of the parton in polarized proton, whether the gluon Sivers function dominates over the quark one or vice versa. Another very promising example is $p^{\uparrow} p \rightarrow J / \psi \gamma X$, which is predominantly initiated by gluongluon scattering (which is an order in $\alpha_{s}$ higher than the gluon contribution in $p^{\uparrow} p \rightarrow \gamma$ jet $X$ ) and for which the color singlet contribution dominates over the color octet one to a large extent $[78,79]$. The same applies to $p^{\uparrow} p \rightarrow J / \psi J / \psi X$ (see the contribution by Lansberg and Shao in this special issue).AFTER@LHC would be very well-suited for studying these processes.

SSA experiments could be done at AFTER@LHC where the beam of protons or lead ions of the LHC would collide with a fixed target that is transversely polarized. Such $p p^{\uparrow}$ and $P b p^{\uparrow}$ collisions would have a center-of-mass energy $\sqrt{s_{N N}}$ of 115 and $72 \mathrm{GeV}$, respectively, and have high luminosity and good coverage in the rapidity region of the transversely polarized target (mid and large $x_{p}^{\uparrow}$ ) [47]. Polarized DrellYan and prompt photon production studies could be done to measure the quark Sivers function very precisely, perhaps to the level that the gluon Sivers function becomes relevant, despite the large values of $x$ in the polarized target. As mentioned $\gamma$ jet and $J / \psi \gamma$ production could be used to study the gluon Sivers effect directly, where the former would need specific selection of the rapidities. In addition, the comparison of $P b p^{\uparrow} \rightarrow \gamma$ jet $X$ and $p p^{\uparrow} \rightarrow$ jjet $X$ would give a further handle on determining the relative sizes of quark and gluon Sivers functions. Other processes, such as $D$-meson or $J / \psi$ production, would allow a similar study of Qiu-Sterman functions, including the trigluon ones, which are of course interesting in their own right. See [28] for a more detailed and quantitative study of twist- 3 transverse single spin asymmetries in proton-proton collisions at the AFTER@LHC experiment. All these possibilities offer a very interesting complementary opportunity or even a competitive alternative to the other existing high-energy particle physics spin projects aiming at studying the role of gluons in transversely polarized protons.

In electron-proton scattering one of the most promising processes to directly probe the gluon Sivers function is open charm production, $e p^{\uparrow} \rightarrow e^{\prime} c \bar{c} X$, which could ideally be studied with an Electron-Ion Collider (EIC). By selecting the charm (or bottom) quark, one effectively eliminates the subprocesses $\gamma^{*} q \rightarrow q g$ and $\gamma^{*} \bar{q} \rightarrow \bar{q} g$ and becomes essentially (this assumes that intrinsic charm contributions are suppressed by selecting sufficiently small- $x$ values) sensitive to $\gamma^{*} g \rightarrow c \bar{c}$ and thus to the gluon Sivers function (a similar 
argument is used in the study of high- $p_{T}$ hadron pairs in muon-deuteron and muon-proton scattering $[80,81]$, where photon-gluon fusion is expected to dominate. The relevant asymmetry $A_{U T}^{\sin \left(\phi_{2 h}-\phi_{S}\right)}$ is found to be $-0.14 \pm 0.15$ (stat.) \pm 0.06 (syst.) at $\left\langle x_{G}\right\rangle=0.13$ for the deuteron and $-0.26 \pm$ 0.09 (stat.) \pm 0.08 (syst.) at $\left\langle x_{G}\right\rangle=0.15$ for the proton. For the interpretation of the data in terms of the gluon Sivers effect, $Q^{2}$ and $p_{T}$ of each hadron need to be sufficiently large to trust factorization). Here the transverse momenta of the heavy quarks are considered to be almost back-toback. There is no problem with TMD factorization breaking contributions of the type discussed in [72], but that does not mean that the process is as straightforward as SIDIS. Even in the case where one considers charm jets, one has to include a description of the transverse momentum distribution inside such a jet. It may be easier to consider $D^{0} \bar{D}^{0}$ measurements (for a study of the twist-3 SSA in large $p_{T} D$ meson production in SIDIS, that is, $e p^{\uparrow} \rightarrow e^{\prime} D X$, see [33, 82]). In either case one deals with 3 TMDs. Such processes involve a different soft factor (in this case a vacuum correlator of 6 Wilson lines) compared to processes involving 2 TMDs as in SIDIS, affecting the predictability. This has been discussed at the one-loop level in [83]. The SSA in $e p^{\uparrow} \rightarrow e^{\prime} D^{0} \bar{D}^{0} X$ has been studied for some models of the gluon Sivers function in [84]; compare Section 2.3.1 of [84]. This may be the "smoking gun" process for the gluonic Sivers effect at an EIC. It should be mentioned though that it actually probes a different gluon Sivers TMD than the hadronic processes discussed above. This is discussed in the next section. It shows that hadronic processes are complementary to DIS processes.

For completeness we mention that when comparing extractions of the gluon Sivers TMD from different processes, one has to take care not only of the process dependence but also of the different energy scales. Under TMD evolution from one scale to another, the transverse momentum distribution changes. For details we refer to [85-90].

\section{Process Dependence of the Gluon Sivers Function}

Once a set of processes that in principle allow probing the gluon Sivers TMD has been obtained, one still has to take into account the fact that such TMD is process-dependent. For quarks the famous overall sign change between the Sivers TMD probed in SIDIS and the one probed in Drell-Yan is expected [3,91-93] and is currently under experimental investigation. For gluons the situation is more complicated as each gluon TMD depends on two gauge links (in the fundamental representation), so there are more possibilities $[11,94,95]$. The gauge link structure of the gluon distributions in $e p \rightarrow e^{\prime} D^{0} \bar{D}^{0} X$ differs from the one in, for instance, $p p \rightarrow \gamma$ jet $X$ (cf. [96] for the comparison at small $x$ ). Clearly, this will complicate the analysis of gluon Sivers effect which will involve more than one gluon Sivers function. In [11] it was demonstrated that any gluon Sivers function can be expressed in terms of two "universal" gluon Sivers functions:

$$
f_{1 T}^{\perp g[U]}\left(x, \mathbf{k}_{\perp}^{2}\right)=\sum_{c=1}^{2} C_{G, c}^{[U]} f_{1 T}^{\perp g(A c)}\left(x, \mathbf{k}_{\perp}^{2}\right),
$$

where the coefficients $C_{G, c}^{[U]}$ are calculable for each partonic subprocess. The first transverse moments of the two distinct gluon Sivers functions are related (at least at tree level) to the two distinct trigluon Qiu-Sterman functions $T_{G}^{(f / d)}$. Therefore, we will refer to the universal gluon Sivers functions as $f_{1 T}^{\perp g(f)}$ and $f_{1 T}^{\perp g(d)}$. The two functions have different behavior under charge conjugation, just like $T_{G}^{(f)}$ is a matrix element of a $C$-even operator and $T_{G}^{(d)}$ of a $C$-odd operator.

The process $e p^{\uparrow} \rightarrow e^{\prime} D^{0} \bar{D}^{0} X$ is dominated by just one partonic subprocess $\gamma g \rightarrow q \bar{q}$ and thus probes the gluon Sivers function with two future-pointing $(+)$ links [97], which is $f_{1 T}^{\perp g(f)}$ [11]. The process $p^{\uparrow} p \rightarrow \gamma$ jet $X$ probes the subprocesses $q g \rightarrow \gamma q$ and $q \bar{q} \rightarrow \gamma g$. If one selects kinematics such that one probes small- $x$ values in the polarized proton, such that $q g \rightarrow \gamma q$ dominates, then this process accesses the gluon Sivers with a future- and past-pointing link, which corresponds to $f_{1 T}^{\perp g(d)}$. The theoretical expectations are different for these two cases.

\section{Theoretical Constraints on Sivers Function}

Constraints on the unintegrated gluon Sivers TMD $f_{1 T}^{\perp g}(x$, $\mathbf{k}_{\perp}^{2}$ ) from fits have to take into account that it is theoretically possible that both the quark and the gluon Sivers TMD can have nodes in $x$ and/or $k_{\perp}[98,99]$. The possibility of a node in $x$ is supported by the observation [35] that the splitting function for $T_{G}^{(f)}$ is negative at small $x$, in analogy to the $\Delta g$ case. Fits to SIDIS data (studied with a rather restrictive parameterization and in a restricted kinematic range) do not appear to require a node [100], but that does not exclude this possibility. Especially when comparing data from different kinematic regions and different processes, this option should be kept in mind. Nodes can of course have a large effect on integrals of Sivers functions, such as the first transverse moment (7) and its first Mellin moment (for parton $a$ )

$$
\left\langle\mathbf{k}_{\perp a}\right\rangle=-M\left(\widehat{\mathbf{S}}_{T} \times \widehat{\mathbf{P}}\right) \int \mathrm{d} x f_{1 T}^{\perp(1) a}(x),
$$

which is the average transverse momentum inside a transversely polarized target. The notation $\left\langle\mathbf{k}_{\perp a}\right\rangle$ comes from [101]. This quantity is related to the Sivers shift [102], the average transverse momentum shift orthogonal to the transverse spin direction, which is normalized to the zeroth transverse moment of the unpolarized TMD $f_{1}^{(0)}(x) \equiv \int \mathrm{d}^{2} k_{\perp} f_{1}\left(x, \mathbf{k}_{\perp}^{2}\right)$ :

$$
\left\langle k_{\perp}^{y}(x)\right\rangle_{U T_{x}}=M \frac{f_{1 T}^{\perp(1)}(x ; \mu, \zeta)}{f_{1}^{(0)}(x ; \mu, \zeta)} .
$$

Here only the $y$-component perpendicular to the transverse spin direction $x$ is nonzero and therefore considered. Note 
that the Sivers shift depends in principle on the renormalization scale $\mu$ and a rapidity variable $\zeta$ but also depends on the path of the gauge link (see below). Furthermore, there is the theoretical issue whether the transverse moments converge. For that reason a regularized version using Bessel moments has been suggested in [102], which for quarks has been evaluated on the lattice in [103]. The lattice calculation of the Sivers shift for $u-d$ is consistent with negative $u$ and positive $d$ Sivers functions in SIDIS, which are expectations that follow from general arguments on final state interactions [104], from a model-dependent relation to GPDs $[105,106]$ (see below) and from fits $[107,108]$.

As said above, $f_{1 T}^{\perp(1) q}(x)$ has a (process-dependent!) relation to the Qiu-Sterman function $T_{q, F}(x, x)$, which so far only has been established at tree level and for quarks. In addition, Burkardt has suggested a model-dependent relation between the integrated quantity $f_{1 T}^{\perp(1)}(x)$ and an integral over the GPD $E\left(x, \xi, \Delta^{2}\right)$ at zero skewness $\xi=0$ (and only for quarks) [105]:

$$
f_{1 T}^{\perp(1)}(x) \propto \int \mathrm{d}^{2} b_{\perp} \overline{\mathscr{I}}\left(\mathbf{b}_{\perp}\right) \frac{\partial}{\partial b_{y}} \mathscr{E}\left(x, \mathbf{b}_{\perp}^{2}\right),
$$

for a nucleon polarized in the transverse $x$ direction. Here $\mathscr{E}\left(x, \mathbf{b}_{\perp}^{2}\right) \equiv \int\left(d^{2} \Delta_{\perp} /(2 \pi)^{2}\right) e^{-i \mathbf{b}_{\perp} \cdot \Delta_{\perp}} E\left(x, 0,-\Delta_{\perp}^{2}\right)$ and $\overline{\mathscr{I}}\left(\mathbf{b}_{\perp}\right)$ is called the lensing function. This relation has been obtained in models $[9,105,106,109]$. It allows relating $\left\langle\mathbf{k}_{\perp q}\right\rangle$ to the anomalous magnetic moment $\kappa_{q}$ associated with the quark $q$ :

$$
\int \mathrm{d} x \int \mathrm{d}^{2} b_{\perp} \mathscr{E}_{q}\left(x, \mathbf{b}_{\perp}^{2}\right)=\kappa_{q},
$$

albeit in a model-dependent and (due to the different integrals involved) only qualitative way. This relation does confirm the expectations for the relative signs between the $u$ and $d$ Sivers functions and has been used in [110] to fit SIDIS data for the Sivers effect with the integral constrained by the anomalous magnetic moments. Interestingly, this led to a new estimation of the quark total angular momentum which turned out to be in agreement with most common GPD extractions [111115]. The relation between $\left\langle\mathbf{k}_{\perp q}\right\rangle$ and $\kappa_{q}$ is also at the heart of the argument by Brodsky and Gardner of why a gluon Sivers function is expected to be small. Using that $\kappa_{u}^{p}=2 \kappa_{p}+\kappa_{n}=$ 1.673 and $\kappa_{d}^{p}=2 \kappa_{n}+\kappa_{p}=-2.033$, one sees the opposite signs reflected, but since $\left|\left(\kappa_{p}+\kappa_{n}\right) / 2\right|=0.06 \ll \kappa_{p / n} \approx 1.8-1.9$ this suggests that there is little room for gluon contributions [60]. If $\left(\kappa_{u}^{p}+\kappa_{d}^{p}\right) / 2$ is taken as a measure for $\kappa_{g}^{p}$, the latter is about $10 \%$ smaller than $\kappa_{q}^{p}$. This would suggest that $\kappa_{g}^{p}$ is of order $1 / N_{c}^{2}$ rather than $1 / N_{c}$, which in turn would suggest a similar additional $1 / N_{c}$ suppression for the gluon Sivers function. Clearly there are various (strong!) assumptions going into this type of argument, such that the conclusion can certainly not be taken at face value. Apart from the assumptions on the relation to the gluon Sivers function, it is not clear that one can use very low-energy quantities to deduce something about the size of the gluon contributions at energies around or above $1 \mathrm{GeV}$ to begin with.

Burkardt derived a further constraint on the fully integrated quantity $\left\langle\mathbf{k}_{\perp a}\right\rangle$, nowadays referred to as the Burkardt sum rule (BSR) [101], stating that the total transverse momentum of all partons in a transversely polarized proton must vanish [116]:

$$
\left\langle\mathbf{k}_{\perp}\right\rangle=\sum_{a=q, \bar{q}, g}\left\langle\mathbf{k}_{\perp a}\right\rangle=\mathbf{0} .
$$

In terms of the Sivers function, the BSR takes the form [59]

$$
\sum_{a=q, \bar{q}, g} \int d x f_{1 T}^{\perp(1) a}(x)=0 .
$$

Its validity has been checked explicitly in a diquark spectator model in [117]. The fits to SIDIS data from [108] at the scale $Q^{2}=2.4 \mathrm{GeV}^{2}$ almost saturate the BSR already with the $u$ and $d$ quark contributions alone:

$$
\begin{aligned}
& \left\langle k_{\perp u}\right\rangle=96_{-28}^{+60} \mathrm{MeV}, \\
& \left\langle k_{\perp d}\right\rangle=-113_{-51}^{+45} \mathrm{MeV} .
\end{aligned}
$$

The contributions of the sea quarks are all small and together allow the following range for the gluon contribution:

$$
-10 \leq\left\langle k_{\perp g}\right\rangle \leq 48 \mathrm{MeV}
$$

This means there is certainly still room for a $30 \%$ contribution from gluons with respect to the valence quarks. Of course, it should be emphasized that these values were obtained under assumptions on the $k_{\perp}$ dependence, the absence of nodes, and extrapolations outside the kinematic region accessed by the SIDIS experiments.

The derivation of the BSR by Burkardt [101] considers gauge links as they appear in SIDIS and involves a gluon correlator containing the antisymmetric $f_{a b c}$ structure constant of $S U(3)$. As shown in $[94,95]$, there is also a gluon correlator with the symmetric $d_{a b c}$ structure constant. As a consequence, inclusion of gauge links in the operator definition of TMD distributions gives rise to two distinct gluon Sivers functions, $f_{1 T}^{\perp g(f)}$ and $f_{1 T}^{\perp g(d)}$ (corresponding to the $(A c)$ label used in [11]). However, the BSR essentially expresses transverse momentum conservation. Since the momentum operator in QCD is $C$-even, only the gluon Sivers function $f_{1 T}^{\perp g(f)}$ which is associated with a $C$-even operator is constrained by the BSR. The gluon Sivers function $f_{1 T}^{\perp g(d)}$, which is associated with a $C$-odd operator, is not expected to satisfy a BSR where quark and gluon contributions cancel each other. Judging from the small- $x$ behavior of $T_{G}^{(d)}(x, x)$ expected from its evolution equation, the integral of $f_{1 T}^{\perp g(d)}$ over $x$ may even not converge.

\section{Conclusions}

In summary, no hard constraints on the size of the gluon Sivers function exist apart from the positivity bound, although the theoretical expectation from large $N_{c}$ considerations (expected to hold approximately for not too small $x$ ) favors a $30 \%$ gluon to quark Sivers ratio, which 
is still completely allowed by all SIDIS data, including the COMPASS deuteron data. It may turn out that the ratio is smaller, but much smaller than $10 \%$ may in turn be considered unnaturally small. Strictly speaking, no direct conclusion about the size of the gluon Sivers function can be drawn from $A_{N}$ data. Like $p^{\uparrow} p \rightarrow \pi X$, many other processes suggested in the literature to probe the gluon Sivers function actually deal with collinear factorization and as such they are sensitive to complicated linear combinations of quarkgluon and trigluon Qiu-Sterman functions (and chiral-odd and fragmentation function versions of them) rather than to Sivers functions directly. Inferring constraints on the gluon Sivers function, even on its large transverse momentum tail, must therefore be done with much care. In the Generalized Parton Model description of $A_{N}$ at midrapidity, the effective gluon Sivers function is currently still allowed to be $30 \%$ of the up quark Sivers function, despite the smallness of the asymmetry. Other suggested processes that in principle probe TMDs may suffer from TMD factorization breaking contributions and any results on the gluon Sivers function from measurements of such processes cannot be trusted. This applies, for instance, to the process $p^{\uparrow} p \rightarrow$ jet jet $X$.

The most promising processes that directly give access to the gluon Sivers effect are $p^{\uparrow} p \rightarrow \gamma$ jet $X, p^{\uparrow} p \rightarrow J / \psi \gamma X$, and $e p^{\uparrow} \rightarrow e^{\prime} c \bar{c} X$. The first process can be studied at RHIC and at a polarized fixed-target experiment at LHC (AFTER@LHC), the second process also at AFTER@LHC, and the third process at a possible future Electron-Ion Collider. Due to the inherent process dependence of TMDs, the gluon Sivers TMD probed is in principle different in these processes. They can be expressed in terms of two universal gluon Sivers functions that appear in different linear combinations in different processes. Extracting and comparing these universal functions is very interesting from a theoretical point of view. The fact that a difference can exist is a consequence of the non-Abelian nature of QCD. Both functions satisfy different theoretical constraints. Although TMD factorization is expected to hold for these processes, that has not been demonstrated yet to all orders. Apart from the process dependence, there is also the issue of modified soft factors to contend with still. Nevertheless, as far as experimentally demonstrating and measuring a gluon Sivers effect in transversely polarized protons, several complementary future possibilities exist, in which AFTER@LHC can play a very important role.

\section{Conflict of Interests}

The authors declare that there is no conflict of interests regarding the publication of this paper.

\section{Acknowledgments}

The authors wish to thank Mauro Anselmino, Maarten Buffing, Umberto D'Alesio, Jean-Philippe Lansberg, Piet Mulders, and Francesco Murgia for useful discussions and/or feedback on the text. C. Lorcé acknowledges support by the Belgian Fund F.R.S.-FNRS via the contract of Chargé de
Recherches. C. Pisano acknowledges support by the "Fonds Wetenschappelijk Onderzoek-Vlaanderen" (FWO) through a postdoctoral Pegasus Marie Curie Fellowship.

\section{References}

[1] D. W. Sivers, "Single-spin production asymmetries from the hard scattering of pointlike constituents," Physical Review D, vol. 41, no. 1, pp. 83-90, 1990.

[2] A. Airapetian, N. Akopov, Z. Akopov et al., "Single-spin asymmetries in semi-inclusive deep-inelastic scattering on a transversely polarized hydrogen target," Physical Review Letters, vol. 94, Article ID 012002, 2005.

[3] J. C. Collins, "Leading-twist single-transverse-spin asymmetries: Drell-Yan and deep-inelastic scattering," Physics Letters B, vol. 536, no. 1-2, pp. 43-48, 2002.

[4] D. Boer and P. J. Mulders, "Time-reversal odd distribution functions in leptoproduction," Physical Review D: Particles, Fields, Gravitation and Cosmology, vol. 57, no. 9, pp. 5780-5786, 1998.

[5] M. Anselmino, M. Boglione, and F. Murgia, "Single spin asymmetry for $p^{\uparrow} p \rightarrow \pi X$ in perturbative QCD," Physics Letters $B$, vol. 362, no. 1-4, pp. 164-172, 1995.

[6] M. Anselmino, M. Boglione, U. D’Alesio, E. Leader, S. Melis, and F. Murgia, "General partonic structure for hadronic spin asymmetries," Physical Review D, vol. 73, no. 1, Article ID 014020, 23 pages, 2006.

[7] A. Bacchetta, U. D’Alesio, M. Diehl, and C. A. Miller, "Singlespin asymmetries: the Trento conventions," Physical Review D, vol. 70, no. 11, Article ID 117504, 2004.

[8] P. J. Mulders and J. Rodrigues, "Transverse momentum dependence in gluon distribution and fragmentation functions," Physical Review D, vol. 63, Article ID 094021, 2001.

[9] S. Meißner, A. Metz, and K. Goeke, "Relations between generalized and transverse momentum dependent parton distributions," Physical Review D, vol. 76, Article ID 034002, 2007.

[10] C. Lorcé and B. Pasquini, "Structure analysis of the generalized correlator of quark and gluon for a spin-1/2 target," Journal of High Energy Physics, vol. 2013, no. 9, article 138, 2013.

[11] M. G. A. Buffing, A. Mukherjee, and P. J. Mulders, "Generalized universality of definite rank gluon transverse momentum dependent correlators," Physical Review D, vol. 88, Article ID 054027, 2013.

[12] J. Collins, Foundations of Perturbative QCD, Cambridge University Press, 2011.

[13] J. Collins, "New definition of tmd parton densities," International Journal of Modern Physics: Conference Series, vol. 4, no. 85, 2011.

[14] M. G. Echevarría, A. Idilbi, and I. Scimemi, "Soft and collinear factorization and transverse momentum dependent parton distribution functions," Physics Letters B, vol. 726, no. 4-5, pp. 795-801, 2013.

[15] M. G. Echevarría, T. Kasemets, P. J. Mulders, and C. Pisano, "QCD evolution of (un)polarized gluon TMDPDFs and the Higgs $q_{T}$-distribution," Journal of High Energy Physics, 2015.

[16] J. Antille, L. Dick, L. Madansky et al., "Spin dependence of the inclusive reaction $\mathrm{p}+\mathrm{p}$ (polarized) $\rightarrow \pi^{0}+\mathrm{X}$ at $24 \mathrm{GeV} / \mathrm{c}$ for high- $p_{T} \pi^{0}$ produced in the central region," Physics Letters B, vol. 94 , no. 4, pp. 523-526, 1980.

[17] D. L. Adams, N. Akchurin, N. I. Belikov et al., "Analyzing power in inclusive $\pi^{+}$and $\pi^{-}$production at high $x_{F}$ with a $200 \mathrm{GeV}$ 
polarized proton beam," Physics Letters B, vol. 264, pp. 462-466, 1991.

[18] D. L. Adams, N. Akchurin, N. I. Belikov et al., "Comparison of spin asymmetries and cross sections in $\pi^{0}$ production by 200 $\mathrm{GeV}$ polarized antiprotons and protons," Physics Letters B, vol. 261, no. 1-2, pp. 201-206, 1991.

[19] K. Krueger, C. Allgower, T. Kasprzyk et al., "Large analyzing power in inclusive $\pi^{ \pm}$production at high $X_{F}$ with a $22-\mathrm{GeV} / \mathrm{c}$ polarized proton beam," Physics Letters B, vol. 459, no. 1-3, pp. 412-416, 1999.

[20] J. Adams, C. Adler, M. M. Aggarwal et al., "Cross sections and transverse single-spin asymmetries in forward neutral-pion production from proton collisions at $\sqrt{s}=200 \mathrm{GeV}$," Physical Review Letters, vol. 92, no. 17, Article ID 171801, 6 pages, 2004.

[21] S. S. Adler, S. Afanasiev, C. Aidala et al., "Measurement of transverse single-spin asymmetries for midrapidity production of neutral pions and charged hadrons in polarized $p+p$ collisions at $\sqrt{s}=200 \mathrm{GeV}$," Physical Review Letters, vol. 95, no. 20, Article ID 202001, 6 pages, 2005.

[22] I. Arsene, I. G. Bearden, D. Beavis et al., "Single-transversespin asymmetries of identified charged hadrons in polarized $p p$ collisions at $\sqrt{s}=62.4 \mathrm{GeV}$," Physical Review Letters, vol. 101, Article ID 042001, 2008.

[23] B. I. Abelev, M. M. Aggarwal, Z. Ahammed et al., "Forward neutral-pion transverse single-spin asymmetries in $p+p$ collisions at $\sqrt{s}=200 \mathrm{GeV}$," Physical Review Letters, vol. 101, no. 22, Article ID 222001, 2008.

[24] L. Adamczyk, G. Agakishiev, M. M. Aggarwal et al., "Transverse single-spin asymmetry and cross section for $\pi^{0}$ and $\eta$ mesons at large Feynman $x$ in $p^{\uparrow}+p$ collisions at $\sqrt{s}=200 \mathrm{GeV}$," Physical Review D, vol. 86, Article ID 051101, 2012.

[25] A. Adare, S. Afanasiev, C. Aidala et al., "Measurement of transverse single-spin asymmetries for $J / \psi$ production in polarized $p+p$ collisions at $\sqrt{s}=200 \mathrm{GeV}$, , Physical Review D, vol. 82, Article ID 112008, 2010, Erratum in Physical Review D, vol. 86, Article ID 099904, 2012.

[26] J. C. Collins, D. E. Soper, and G. Sterman, "Factorization of hard processes in QCD," Advanced Series on Directions in High Energy Physics, vol. 5, pp. 1-9, 1988.

[27] M. Anselmino, U. D’Alesio, and S. Melis, “Transverse single-spin asymmetries in proton-proton collisions at the AFTER@LHC experiment in a TMD factorisation scheme," http://arxiv.org/abs/1504.03791.

[28] K. Kanazawa, Y. Koike, A. Metz, and D. Pitonyak, “Transverse single-spin asymmetries in proton-proton collisions at the AFTER@LHC experiment," http://arxiv.org/abs/1502.04021.

[29] M. Luo, J. Qiu, and G. Sterman, ITP-SB-91-56, C91-08-18.

[30] J. Qiu and G. Sterman, "Single transverse spin asymmetries," Physical Review Letters, vol. 67, no. 17, pp. 2264-2267, 1991.

[31] J. Qiu and G. Sterman, "Single transverse-spin asymmetries in hadronic pion production," Physical Review D, vol. 59, Article ID 014004, 1998.

[32] X. D. Ji, "Gluon correlations in the transversely polarized nucleon," Physics Letters B, vol. 289, no. 1-2, pp. 137-142, 1992.

[33] Z. B. Kang and J. W. Qiu, "Single transverse-spin asymmetry for $D$-meson production in semi-inclusive deep inelastic scattering," Physical Review D, vol. 78, no. 3, Article ID 034005, 11 pages, 2008.

[34] Z. B. Kang, J. W. Qiu, W. Vogelsang, and F. Yuan, "Accessing trigluon correlations in the nucleon via the single spin asymmetry in open charm production," Physical Review D, vol. 78, no. 11, Article ID 114013, 9 pages, 2008.
[35] A. Schäfer and J. Zhou, "A note on the scale evolution of trigluon correlations," http://arxiv.org/abs/1308.4961.

[36] D. Boer, P. J. Mulders, and F. Pijlman, "Universality of Todd effects in single spin and azimuthal asymmetries," Nuclear Physics B, vol. 667, no. 1-2, pp. 201-241, 2003.

[37] X. Ji, J. W. Qiu, W. Vogelsang, and F. Yuan, "Unified picture for single transverse-spin asymmetries in hard-scattering processes," Physical Review Letters, vol. 97, Article ID 082002, 2006.

[38] V. M. Braun, A. N. Manashov, and B. Pirnay, "Scale dependence of twist-three contributions to single spin asymmetries," Physical Review D, vol. 80, Article ID 114002, 2009, Erratum in: Physical Review D, vol. 86, Article ID 119902, 2012.

[39] A. Schäfer and J. Zhou, "Transverse single spin asymmetry in hadronic $\eta_{c, b}$ production," Physical Review D, vol. 88 , Article ID 014008, 2013.

[40] K. Kanazawa and Y. Koike, "New analysis of the single transverse-spin asymmetry for hadron production at RHIC," Physical Review D, vol. 82, no. 3, Article ID 034009, 12 pages, 2010.

[41] K. Kanazawa and Y. Koike, "A phenomenological study of single transverse-spin asymmetry for inclusive light-hadron productions at RHIC," Physical Review D, vol. 83, Article ID 114024, 2011.

[42] K. Kanazawa, Y. Koike, A. Metz, and D. Pitonyak, “Towards an explanation of transverse single-spin asymmetries in protonproton collisions: the role of fragmentation in collinear factorization," Physical Review D, vol. 89, no. 11, Article ID 111501, 5 pages, 2014.

[43] A. Adare, S. Afanasiev, C. Aidala et al., "Measurement of transverse-single-spin asymmetries for midrapidity and forward-rapidity production of hadrons in polarized $p+p$ collisions at $\sqrt{s}=200$ and $62.4 \mathrm{GeV}$,' Physical Review D, vol. 90, Article ID 012006, 2014.

[44] H. Beppu, K. Kanazawa, Y. Koike, and S. Yoshida, “Three-gluon contribution to the single spin asymmetry for light hadron production in $p p$ collision," Physical Review D, vol. 89, Article ID 034029, 2014.

[45] H. Liu, "Transverse single spin asymmetry in heavy flavor production in polarized $p+p$ collisions at PHENIX," AIP Conference Proceedings, vol. 1149, no. 1, pp. 439-442, 2009.

[46] Y. Koike and S. Yoshida, "Probing the three-gluon correlation functions by the single spin asymmetry in $p^{\uparrow} p \rightarrow D X$," Physical Review D, vol. 84, Article ID 014026, 2011.

[47] S. J. Brodsky, F. Fleuret, C. Hadjidakis, and J. P. Lansberg, "Physics opportunities of a fixed-target experiment using LHC beams," Physics Reports, vol. 522, no. 4, pp. 239-255, 2013.

[48] U. D’Alesio, F. Murgia, and C. Pisano, "Towards a first estimate of the gluon Sivers function from $A_{N}$ data in $p p$ collisions at RHIC," http://arxiv.org/abs/1506.03078.

[49] M. Anselmino, U. D’Alesio, S. Melis, and F. Murgia, "Constraints on the gluon Sivers distribution via transverse single spin asymmetries at midrapidity in $p^{\uparrow} p \rightarrow \pi^{0} X$ processes at BNL RHIC," Physical Review D, vol. 74, no. 9, Article ID 094011, 7 pages, 2006.

[50] A. Airapetian, N. Akopov, Z. Akopov et al., "Observation of the naive-T-odd sivers effect in deep-inelastic scattering," Physical Review Letters, vol. 103, Article ID 152002, 2009.

[51] C. Adolph, M. G. Alekseev, V. Y. Alexakhin et al., "II-experimental investigation of transverse spin asymmetries in $\mu$-p SIDIS processes: sivers asymmetries," Physics Letters B, vol. 717, no. 4-5, pp. 383-389, 2012. 
[52] X. Qian, K. Allada, C. Dutta et al., "Single spin asymmetries in charged pion production from semi-inclusive deep inelastic scattering on a transversely polarized ${ }^{3} \mathrm{He}$ target at $Q^{2}=1.4-2.7$ $\mathrm{GeV}^{2}$," Physical Review Letters, vol. 107, Article ID 072003, 2011.

[53] Y. X. Zhao, Y. Wang, K. Allada et al., "Single spin asymmetries in charged kaon production from semi-inclusive deep inelastic scattering on a transversely polarized ${ }^{3} \mathrm{He}$ target," Physical Review C, vol. 90, no. 5, Article ID 055201, 6 pages, 2014.

[54] M. Alekseev, A. Amoroso, and V. Y. Alexakhin, "Collins and Sivers asymmetries for pions and kaons in muon-deuteron DIS," Physics Letters B, vol. 673, no. 2, pp. 127-135, 2009.

[55] M. Anselmino, M. Boglione, U. D’Alesio, S. Melis, F. Murgia, and A. Prokudin, "Sivers distribution functions and the latest SIDIS data," http://arxiv.org/abs/1107.4446.

[56] P. V. Pobylitsa, "Transverse-momentum dependent parton distributions in large-Nc QCD," http://arxiv.org/abs/hep-ph/ 0301236.

[57] A. Drago, "Time-reversal-odd distribution functions in chiral models with vector mesons," Physical Review D, vol. 71, no. 5, Article ID 057501, 4 pages, 2005.

[58] A. V. Efremov, K. Goeke, and P. V. Pobylitsa, "Gluon and quark distributions in large N(c) QCD: theory versus phenomenology," Physics Letters, Section B: Nuclear, Elementary Particle and High-Energy Physics, vol. 488, no. 2, pp. 182-186, 2000.

[59] A. V. Efremov, K. Goeke, S. Menzel, A. Metz, and P. Schweitzer, "Sivers effect in semi-inclusive DIS and in the Drell-Yan process," Physics Letters B, vol. 612, no. 3-4, pp. 233-244, 2005.

[60] S. J. Brodsky and S. Gardner, "Evidence for the absence of gluon orbital angular momentum in the nucleon," Physics Letters B, vol. 643, no. 1, pp. 22-28, 2006.

[61] A. Airapetian, N. Akopov, Z. Akopov et al., "Transverse target single-spin asymmetry in inclusive electroproduction of charged pions and kaons," Physics Letters B, vol. 728, pp. 183190, 2014.

[62] K. Allada, Y. X. Zhao, K. Aniol et al., "Single spin asymmetries of inclusive hadrons produced in electron scattering from a transversely polarized ${ }^{3} \mathrm{He}$ target," Physical Review C, vol. 89, Article ID 042201, 2014.

[63] Z. B. Kang, A. Metz, J. W. Qiu, and J. Zhou, "Exploring the structure of the proton through polarization observables in $l_{p} \rightarrow$ jetX," Physical Review D, vol. 84, Article ID 034046, 2011.

[64] D. Boer and W. Vogelsang, "Asymmetric jet correlations in $p p^{\uparrow}$ scattering," Physical Review D, vol. 69, Article ID 094025, 2004.

[65] M. Anselmino, M. Boglione, U. D’Alesio, E. Leader, and F. Murgia, "Accessing Sivers gluon distribution via transverse single spin asymmetries in $p \uparrow p \rightarrow D X$ processes at BNL RHIC," Physical Review D-Particles, Fields, Gravitation and Cosmology, vol. 70, no. 7, Article ID 74025, 2004.

[66] I. Schmidt, J. Soffer, and J. J. Yang, "Transverse single spin asymmetries in photon production," Physics Letters B, vol. 612, no. 3-4, pp. 258-262, 2005.

[67] A. Bacchetta, C. Bomhof, U. D’Alesio, P. J. Mulders, and F. Murgia, "Sivers single-spin asymmetry in photon-jet production," Physical Review Letters, vol. 99, no. 21, Article ID 212002, 2007.

[68] L. Adamczyk, G. Agakishiev, M. M. Aggarwal et al., "Longitudinal and transverse spin asymmetries for inclusive jet production at mid-rapidity in polarized $p+p$ collisions at $\sqrt{s}=200 \mathrm{GeV}$," Physical Review D, vol. 86, p. 032006, 2012.

[69] L. C. Bland, E. J. Brash, H. J. Crawford et al., "Cross sections and transverse single-spin asymmetries in forward jet production from proton collisions at $\sqrt{s}=500 \mathrm{GeV}$,' http://arxiv.org/abs/ 1304.1454.

[70] M. Anselmino, M. Boglione, U. D’Alesio, S. Melis, F. Murgia, and A. Prokudin, "Sivers effect and the single spin asymmetry AN in $p^{\uparrow} p \rightarrow h X$ processes," Physical Review D: Particles, Fields, Gravitation and Cosmology, vol. 88, no. 5, Article ID 054023, 2013.

[71] U. D'Alesio, F. Murgia, and C. Pisano, "Azimuthal asymmetries for hadron distributions inside a jet in hadronic collisions," Physical Review D-Particles, Fields, Gravitation and Cosmology, vol. 83, no. 3, Article ID 034021, 2011.

[72] T. C. Rogers and P. J. Mulders, "No generalized transverse momentum dependent factorization in the hadroproduction of high transverse momentum hadrons," Physical Review D, vol. 81, no. 9, Article ID 094006, 17 pages, 2010.

[73] B. I. Abelev, M. M. Aggarwal, Z. Ahammed et al., "Measurement of transverse single-spin asymmetries for Dijet production in proton-proton collisions at $\sqrt{s}=200 \mathrm{GeV}$," Physical Review Letters, vol. 99, Article ID 142003, 2007.

[74] C. J. Bomhof, P. J. Mulders, W. Vogelsang, and F. Yuan, "Singletransverse spin asymmetry in dijet correlations at hadron colliders," Physical Review D, vol. 75, no. 7, Article ID 074019, 2007.

[75] J. W. Qiu, W. Vogelsang, and F. Yuan, "Single transverse-spin asymmetry in hadronic dijet production," Physical Review D, vol. 76, Article ID 074029, 2007.

[76] W. Vogelsang and F. Yuan, "Hadronic dijet imbalance and transverse-momentum-dependent parton distributions," Physical Review D, vol. 76, no. 9, Article ID 094013, 6 pages, 2007.

[77] S. Catani, M. Grazzini, and A. Torre, "Transverse-momentum resummation for heavy-quark hadroproduction," Nuclear Physics B, vol. 890, pp. 518-538, 2015.

[78] W. J. den Dunnen, J.-P. Lansberg, C. Pisano, and M. Schlegel, "Accessing the transverse dynamics and polarization of gluons inside the proton at the LHC," Physical Review Letters, vol. 112, no. 21, Article ID 212001, 2014.

[79] J. P. Lansberg, M. Anselmino, R. Arnaldi et al., "Spin physics and TMD studies at a fixed-target experiment at the LHC (AFTER@LHC),"EPJ Web of Conferences, vol. 85, Article ID 02038, 6 pages, 2015.

[80] A. Szabelski, "Gluon contribution to the Sivers effect. COMPASS results on deuteron target," EPJ Web of Conferences, vol. 85, Article ID 02006, 6 pages, 2015.

[81] A. Szabelski and COMPASS Collaboration, "Results of the gluon Sivers asymmetry extraction from COMPASS data with transversely polarised targets," in Proceedings of the 7th International Conference on Quarks and Nuclear Physics (QNP '15), Valparaiso, Chile, March 2015, http://wwwcompass.cern .ch/compass/publications/talks/t2015/szabelski_qnp2015.pdf.

[82] H. Beppu, Y. Koike, K. Tanaka, and S. Yoshida, "Single transverse-spin asymmetry in large $P_{T}$ open charm production at an electron-ion collider," Physical Review D, vol. 85, Article ID 114026, 2012.

[83] R. Zhu, P. Sun, and F. Yuan, "Low transverse momentum heavy quark pair production to probe gluon tomography," Physics Letters B, vol. 727, no. 4-5, pp. 474-479, 2013.

[84] D. Boer, M. Diehl, R. Milner et al., "Gluons and the quark sea at high energies: distributions, polarization, tomography," http://arxiv.org/abs/1108.1713.

[85] Z. B. Kang, B. W. Xiao, and F. Yuan, "QCD resummation for single spin asymmetries," Physical Review Letters, vol. 107, Article ID 152002, 2011. 
[86] S. M. Aybat, J. C. Collins, J. W. Qiu, and T. C. Rogers, "QCD evolution of the Sivers function," Physical Review D: Particles, Fields, Gravitation and Cosmology, vol. 85, no. 3, Article ID 034043, 2012.

[87] M. Anselmino, M. Boglione, and S. Melis, "Strategy towards the extraction of the Sivers function with transverse momentum dependent evolution," Physical Review D, vol. 86, no. 1, Article ID 014028, 2012.

[88] P. Sun and F. Yuan, "Energy evolution for the Sivers asymmetries in hard processes," Physical Review D, vol. 88, p. 034016, 2013.

[89] D. Boer, "TMD evolution of the Sivers asymmetry," Nuclear Physics B, vol. 874, no. 1, pp. 217-229, 2013.

[90] M. G. Echevarría, A. Idilbi, Z. B. Kang, and I. Vitev, "QCD evolution of the Sivers asymmetry," Physical Review D, vol. 89, no. 7, Article ID 074013, 2014.

[91] S. J. Brodsky, D. S. Hwang, and I. Schmidt, "Final-state interactions and single-spin asymmetries in semi-inclusive deep inelastic scattering," Physics Letters B, vol. 530, no. 1-4, pp. 99107, 2002.

[92] S. J. Brodsky, D. S. Hwang, and I. Schmidt, "Initial-state interactions and single-spin asymmetries in Drell-Yan processes," Nuclear Physics B, vol. 642, no. 1-2, pp. 344-356, 2002.

[93] A. V. Belitsky, X. Ji, and F. Yuan, "Final state interactions and gauge invariant parton distributions," Nuclear Physics B, vol. 656, no. 1-2, pp. 165-198, 2003.

[94] C. J. Bomhof and P. J. Mulders, "Gluonic pole cross sections and single spin asymmetries in hadron-hadron scattering," Journal of High Energy Physics, vol. 2007, no. 2, article 029, 2007.

[95] C. J. Bomhof and P. J. Mulders, "Non-universality of transverse momentum dependent parton distribution functions," Nuclear Physics B, vol. 795, no. 1-2, pp. 409-427, 2008.

[96] F. Dominguez, C. Marquet, B. W. Xiao, and F. Yuan, "Universality of unintegrated gluon distributions at small $x$," Physical Review D, vol. 83, no. 10, Article ID 105005, 27 pages, 2011.

[97] C. Pisano, D. Boer, S. J. Brodsky, M. G. A. Buffing, and P. J. Mulders, "Linear polarization of gluons and photons in unpolarized collider experiments," Journal of High Energy Physics, vol. 2013, no. 10, article 024, 2013.

[98] D. Boer, "On a possible node in the Sivers and Qiu-Sterman functions," Physics Letters B, vol. 702, no. 4, pp. 242-245, 2011.

[99] Z. B. Kang, J. W. Qiu, W. Vogelsang, and F. Yuan, "Observation concerning the process dependence of the Sivers functions," Physical Review D, vol. 83, no. 9, Article ID 094001, 9 pages, 2011.

[100] Z. B. Kang and A. Prokudin, "Global fitting of single spin asymmetry: an attempt," Physical Review D, vol. 85, Article ID 074008, 2012.

[101] M. Burkardt, "Sivers mechanism for gluons," Physical Review D, vol. 69, no. 9, Article ID 091501, 4 pages, 2004.

[102] D. Boer, L. Gamberg, B. Musch, and A. Prokudin, "Besselweighted asymmetries in semi-inclusive deep inelastic scattering," Journal of High Energy Physics, vol. 2011, no. 10, article 021, 2011.

[103] B. U. Musch, P. Hägler, M. Engelhardt, J. W. Negele, and A. Schäfer, "Sivers and Boer-Mulders observables from lattice QCD," Physical Review D: Particles, Fields, Gravitation and Cosmology, vol. 85, Article ID 094510, 2012.

[104] M. Burkardt, "Impact parameter dependent parton distributions and transverse single spin asymmetries," Physical Review $D$, vol. 66, Article ID 114005, 2002.

[105] M. Burkardt, "Chromodynamic lensing and transverse single spin asymmetries," Nuclear Physics A, vol. 735, no. 1-2, pp. 185199, 2004.
[106] M. Burkardt and D. S. Hwang, "Sivers effect and generalized parton distributions in impact parameter space," Physical Review D, vol. 69, Article ID 074032, 2004.

[107] M. Anselmino, M. Boglione, U. D’Alesio, A. Kotzinian, F. Murgia, and A. Prokudin, "Extracting the Sivers function from polarized semi-inclusive deep inelastic scattering data and making predictions," Physical Review D, vol. 72, Article ID 094007, 2005, Erratum in: Physical Review D, vol. 72, Article ID 099903, 2005.

[108] M. Anselmino, M. Boglione, U. D’Alesio et al., "Sivers effect for pion and kaon production in semi-inclusive deep inelastic scattering," The European Physical Journal A, vol. 39, no. 1, pp. 89-100, 2009.

[109] L. Gamberg and M. Schlegel, "Final state interactions and the transverse structure of the pion using non-perturbative eikonal methods," Physics Letters B, vol. 685, no. 1, pp. 95-103, 2010.

[110] A. Bacchetta and M. Radici, "Constraining quark angular momentum through semi-inclusive measurements," Physical Review Letters, vol. 107, Article ID 212001, 2011.

[111] M. Guidal, M. V. Polyakov, A. V. Radyushkin, and M. Vanderhaeghen, "Nucleon form factors from generalized parton distributions," Physical Review D-Particles, Fields, Gravitation and Cosmology, vol. 72, no. 5, Article ID 054013, 2005.

[112] M. Diehl, T. Feldmann, R. Jakob, and P. Kroll, "Generalized parton distributions from nucleon form factor data," European Physical Journal C, vol. 39, no. 1, 2005.

[113] S. Ahmad, H. Honkanen, S. Liuti, and S. K. Taneja, "Generalized parton distributions from hadronic observables: zero skewness," Physical Review D, vol. 75, no. 9, Article ID 094003, 19 pages, 2007.

[114] S. V. Goloskokov and P. Kroll, "The target asymmetry in hard vector-meson electroproduction and parton angular momenta," European Physical Journal C, vol. 59, no. 4, pp. 809-819, 2009.

[115] M. Diehl and P. Kroll, "Nucleon form factors, generalized parton distributions and quark angular momentum," The European Physical Journal C, vol. 73, article 2397, 2013.

[116] C. Lorcé, “The light-front gauge-invariant energy-momentum tensor," http://xxx.tau.ac.il/abs/1502.06656.

[117] K. Goeke, S. Meissner, A. Metz, and M. Schlegel, "Checking the Burkardt sum rule for the Sivers function by model calculations," Physics Letters B, vol. 637, no. 4-5, pp. 241-244, 2006. 

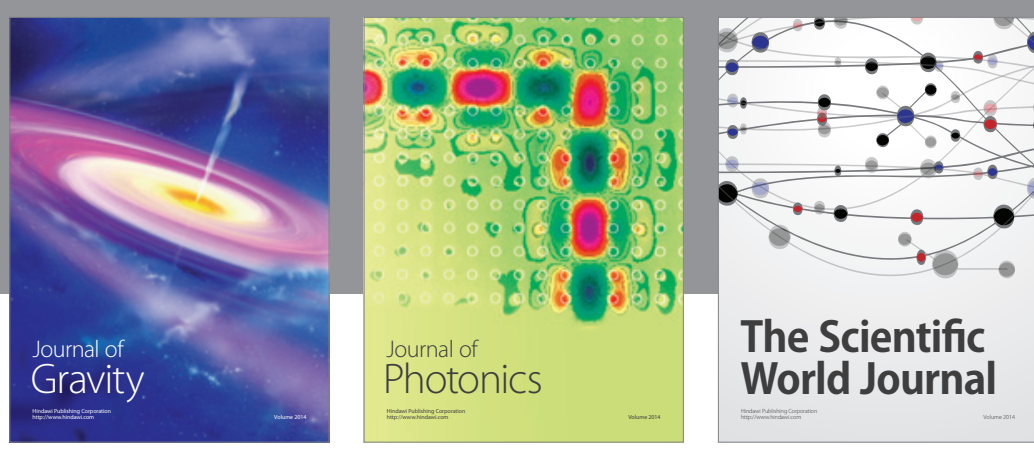

The Scientific World Journal
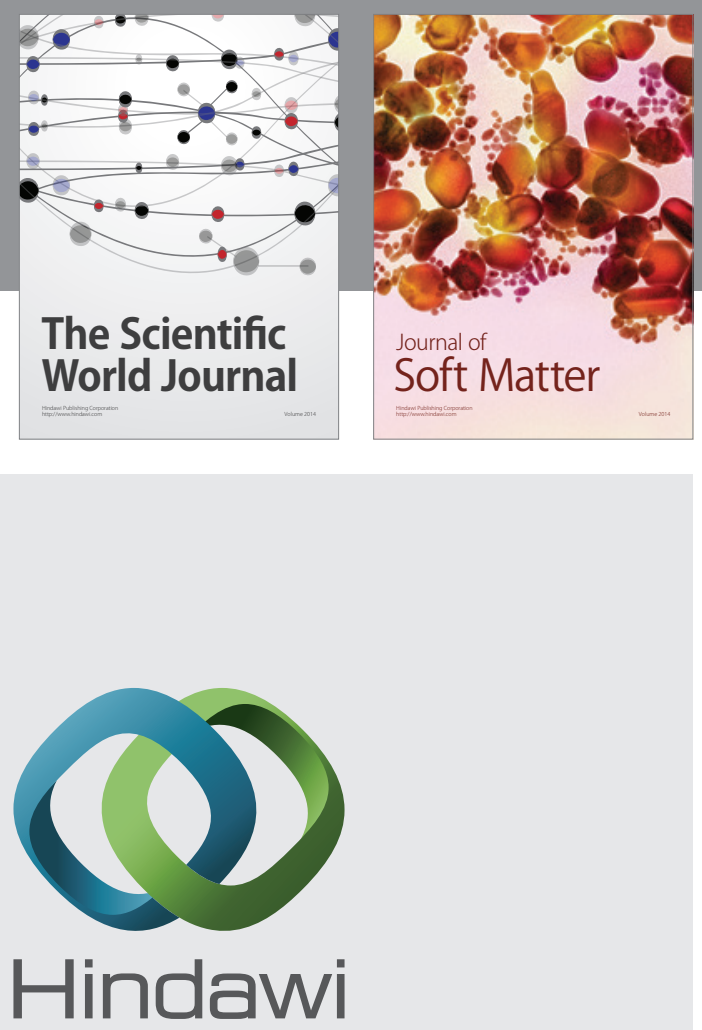

Submit your manuscripts at

http://www.hindawi.com

nternational Journal of

Statistical Mechanics
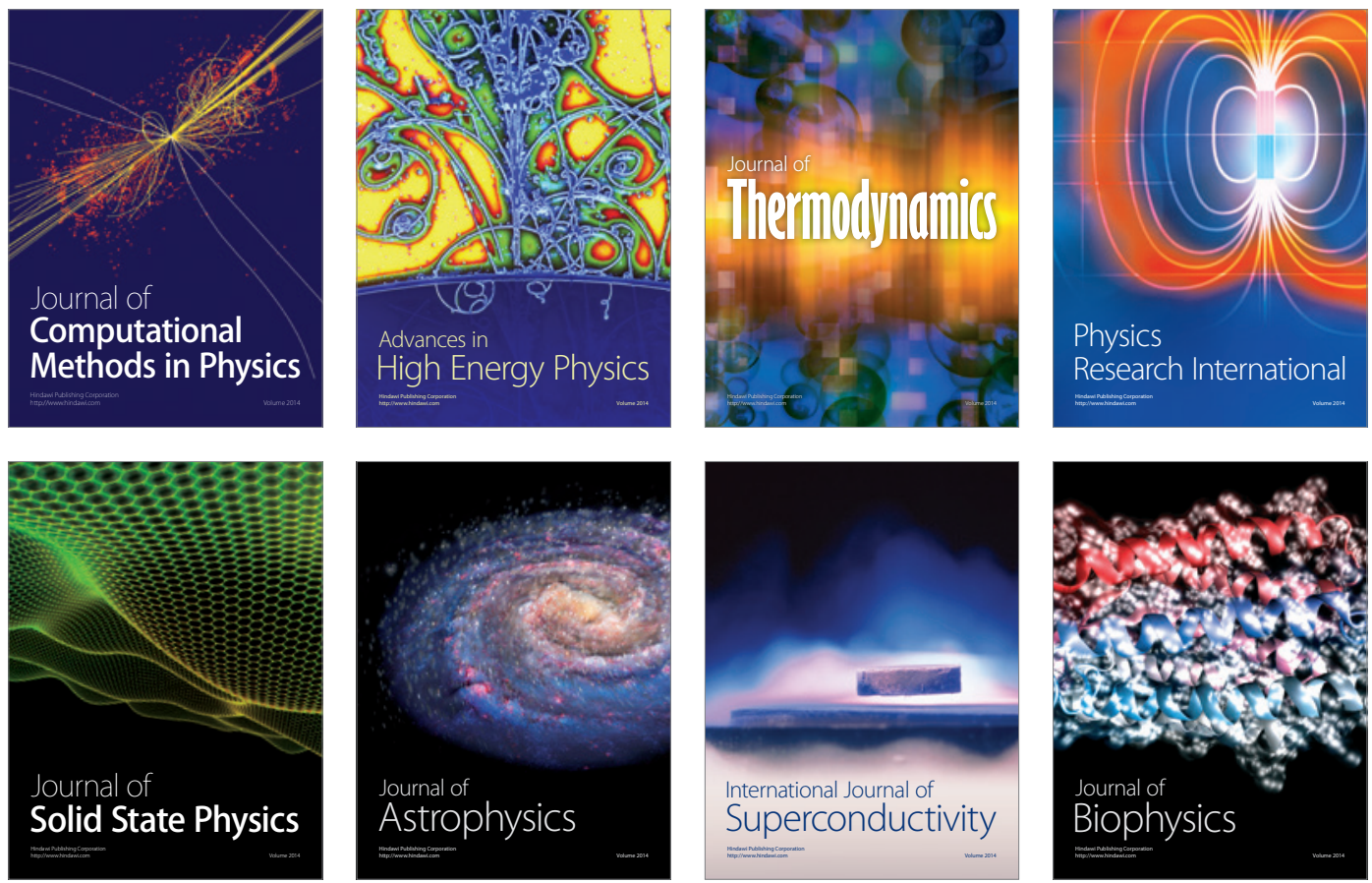
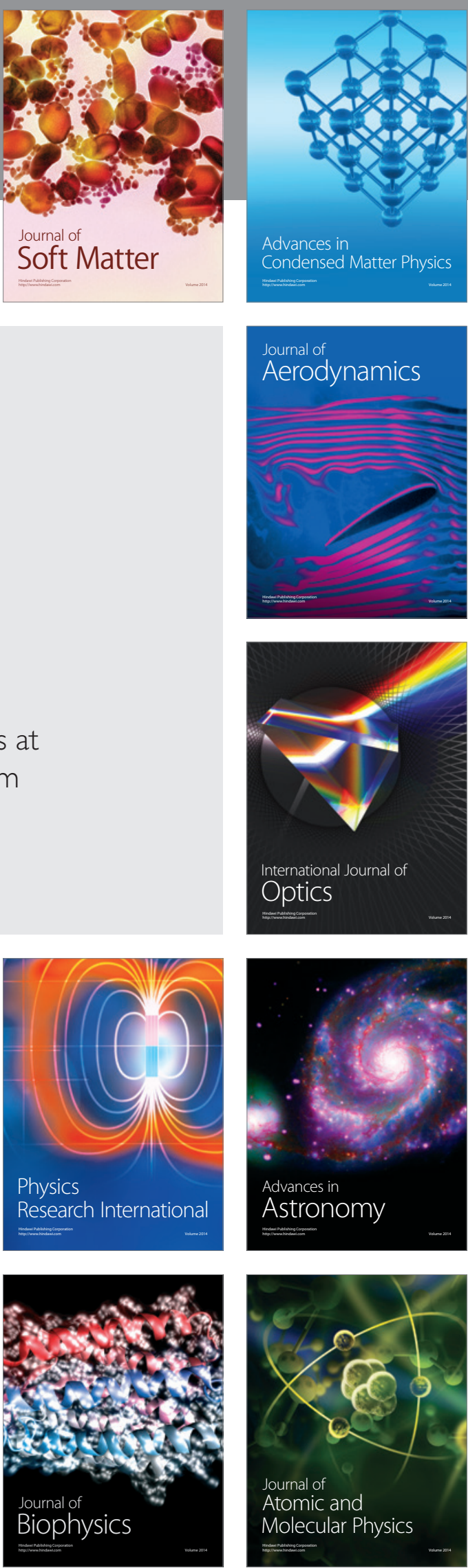